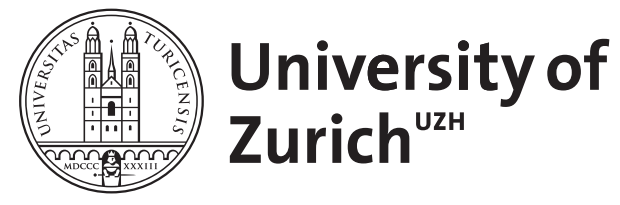

Ultrasound diagnosis of a diaphragmatic hernia in a 23-year-old Hanoverian gelding

\author{
Kaupp, G ; Mitchell, Katharyn J
}

DOI: https://doi.org/10.17236/sat00117

Posted at the Zurich Open Repository and Archive, University of Zurich ZORA URL: https://doi.org/10.5167/uzh-146209

Journal Article

Published Version

Originally published at:

Kaupp, G; Mitchell, Katharyn J (2017). Ultrasound diagnosis of a diaphragmatic hernia in a 23-year-old Hanoverian gelding. Schweizer Archiv für Tierheilkunde, 159(5):301-304.

DOI: https://doi.org/10.17236/sat00117 


\title{
Ultrasound diagnosis of a diaphragmatic hernia in a 23-year-old Hanoverian gelding
}

\author{
G. Kaupp ${ }^{1}$, K.J. Mitchell ${ }^{1}$ \\ ${ }^{1}$ Equine Department, Vetsuisse Faculty, University of Zurich
}

\section{Introduction}

A definitive pre-surgical diagnosis of a diaphragmatic hernia can be challenging in horses. The clinical signs are diverse and horses can present with respiratory compromise, exercise intolerance, lethargy, weight loss, mild or intermittent colic signs, with acute onset of severe colic being the most common sign.

While rectal and auscultatory findings may suggest a diaphragmatic hernia (an 'empty' feeling abdomen on rectal palpation, partially absent bronchovesicular sounds or borborygmi audible during cardiothoracic auscultation), a final diagnosis was historically only achieved during exploratory laparotomy or necropsy (Hart and Brown, 2009; Romero and Rodgerson, 2010). Gas-filled intestine within the thoracic cavity is pathognomonic for a diaphragmatic hernia (Hassel, 2007) however thoracic and abdominal radiography in adult horses is often not practical or available in a field setting.

Transabdominal ultrasonography is readily available for most clinicians. It is quick and easy to perform in horses and is non-invasive. The diaphragm can be visualized on both sides of the chest, lying between the lung and the liver (right) or spleen and stomach (left) (Rantanen, 1986). The presence of free fluid and gastrointestinal organs within the pleural or pericardial space with discontinuity of the diaphragm is characteristic for a diaphragmatic hernia (Williams et al., 1998).

This short communication describes the use of ultrasonography to diagnose and monitor a diaphragmatic hernia in a horse with a history of intermittent mild colic and weight-loss.

\section{Case History}

A 23-year-old Hanoverian gelding presented with a history of weight loss over the previous 4 weeks initial- ly associated with an episode of colic and inappetence. A second episode of mild colic with lethargy, recumbency and inappetence occurred one week prior to presentation. The horse sweated heavily and coughed occasionally during both colic episodes but responded to treatment with nonsteroidal anti-inflammatory drugs.

\section{Clinical Findings}

On presentation, the horse was bright and alert. Body condition score was $3.5 / 9$ and the horse weighed $550 \mathrm{~kg}$. Temperature and heart rate were within normal limits and auscultation of the heart and gastrointestinal sounds was unremarkable. Respiratory rate was slightly elevated (24 breaths/min) with mildly increased abdominal effort during expiration. On auscultation the bronchovesicular lung sounds were prominent dorsally with quieter than expected regions cranioventrally in each hemithorax. A complete blood count and chemistry panel was unremarkable.

Ultrasonography of the thorax and abdomen revealed mild bilateral hypoechoic pleural effusion with both small and large intestinal structures visible within the pleural space between the lung and pericardium in the right ventral thorax (Fig. 1A). The entrapped small intestine showed motility, moving in and out of the field of view. Between intercostal spaces (ICS) 8, 9 and 10 on the right side, a discontinuity of the diaphragm was observed, with the diaphragm displaced off the body wall and the ventral diaphragmatic tissue appearing thickened and wavy compared to the continuous diaphragm on the left side (Fig. 1B). A small area of liver was visualized in the right dorso-caudal abdomen and the hepatic vasculature was more prominent than normal. The stomach appeared enlarged with the caudal border visible in the $15^{\text {th }}$ ICS on the left side. At this time, all the visualized intra-abdominal small and large intestine had appropriate wall thickness and motility. A https://doi.org/ 10.17236/sat00117

Received: 28.07.2016 Accepted: 20.10.2016 
Ultrasound diagnosis of diaphragmatic hernia in a 23-year-old Hanoverian gelding

G. Kaupp, K.J. Mitchell
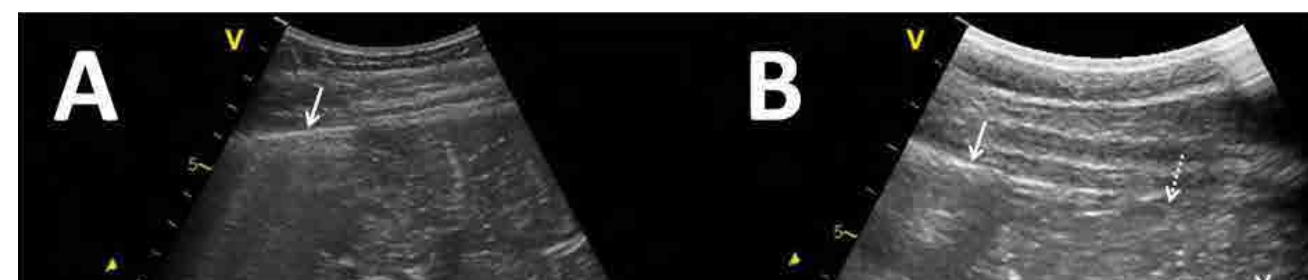

$+5$
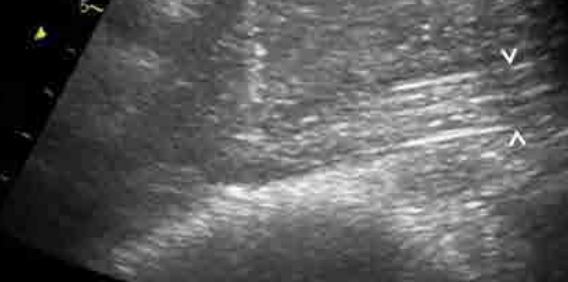

2
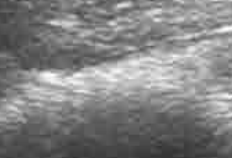

.

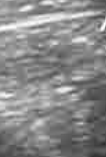

Fig. 1: Ultrasonographic images of several distended small intestinal loops directly adjacent and ventral to the right lung lobe (arrow) with no diaphragm visible in the normally expected location between those structures (A) and showing a discontinuity of the diaphragm (diaphragm indicated with arrowheads) (B). The diaphragm is diverging from the body wall, thus not in its expected anatomic position. Small intestinal loops (dashed arrow) can be seen directly adjacent to the right lung lobe (arrow) and dorsal to the diaphragm. Images obtained from the right cranio-ventral thorax at ICS 6 (A) and from the right middle thorax at ICS 9 (B). Dorsal is to the left. Scan depth is indicated by the scale to the left (in centimeters).

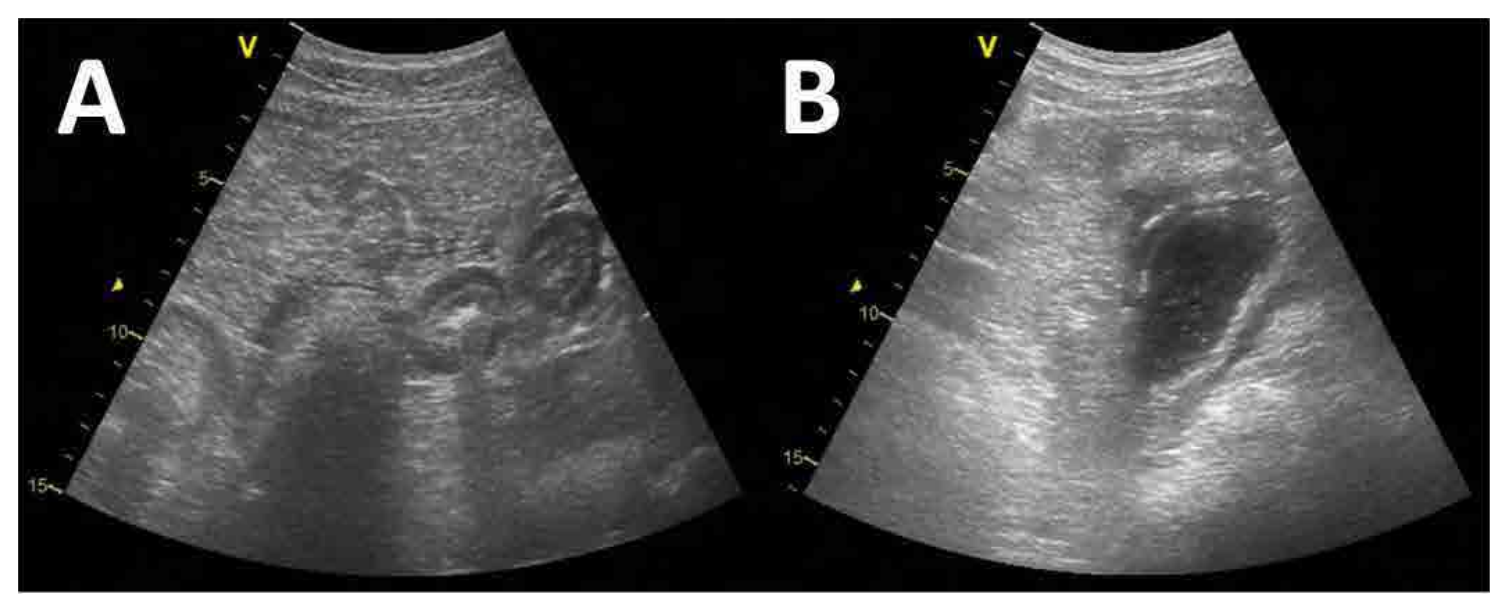

Fig. 2: Ultrasonographic images of several small intestinal loops with marked wall thickening (A), and a single distended and amotile loop of small intestine (B) likely due to temporary obstruction or incarceration within the hernia. Images obtained from the right caudo-ventral abdomen. Dorsal is to the left. Scan depth is indicated by the scale to the left (in centimeters).

mild amount of hypoechoic peritoneal fluid was observed ventrally.

Thoracic radiographs showed an increased opacity of the lung with thickened bronchial walls consistent with a mild generalized bronchointerstitial pattern and an undulating diaphragm, considered to be caused by gas in the stomach lying against the diaphragm. Radiographically no pleural effusion was visible, with no evidence of gastrointestinal organs located within the thorax. Rectal examination was unremarkable. To determine the viability of the entrapped intestine, pleuraland abdominocentesis was performed. Both the pleural and abdominal fluid were similar; yellow, slightly turbid, with a low lactate concentration $(<0.7 \mathrm{mmol} / \mathrm{l})$, total protein (TP) of $<20 \mathrm{~g} / \mathrm{L}$ and a nucleated cell count (NCC) of $<1200 / \mu 1$ consistent with a transudate.
On repeat ultrasound the following day, the right sided diaphragmatic discontinuity was reproducible and the pleural effusion unchanged, however no intestinal structures were clearly visible in the right hemi-thorax. In the right caudo-ventral abdomen several loops of small intestine with a wall thickness of $7 \mathrm{~mm}$ (normal $<4 \mathrm{~mm}$ ) and sluggish motility were observed (Fig. 2A and B). In the right middle abdomen a region of dorsal colon or caecum was visualized with a wall thickness of $10 \mathrm{~mm}$ (normal $<4 \mathrm{~mm}$ ). The liver was not visible in the normal location on the right side, with only the lung, diaphragm and presumed dorsal colon observed from ICS 8-15. It was suspected that the abdominal organs were able to move freely in and out of the diaphragmatic rent, with the previous colic episodes related to temporary obstruction or incarceration of intestine within the hernia. Explorative laparotomy was declined due to the horses' age, poor prognosis for a successful surgical outcome 


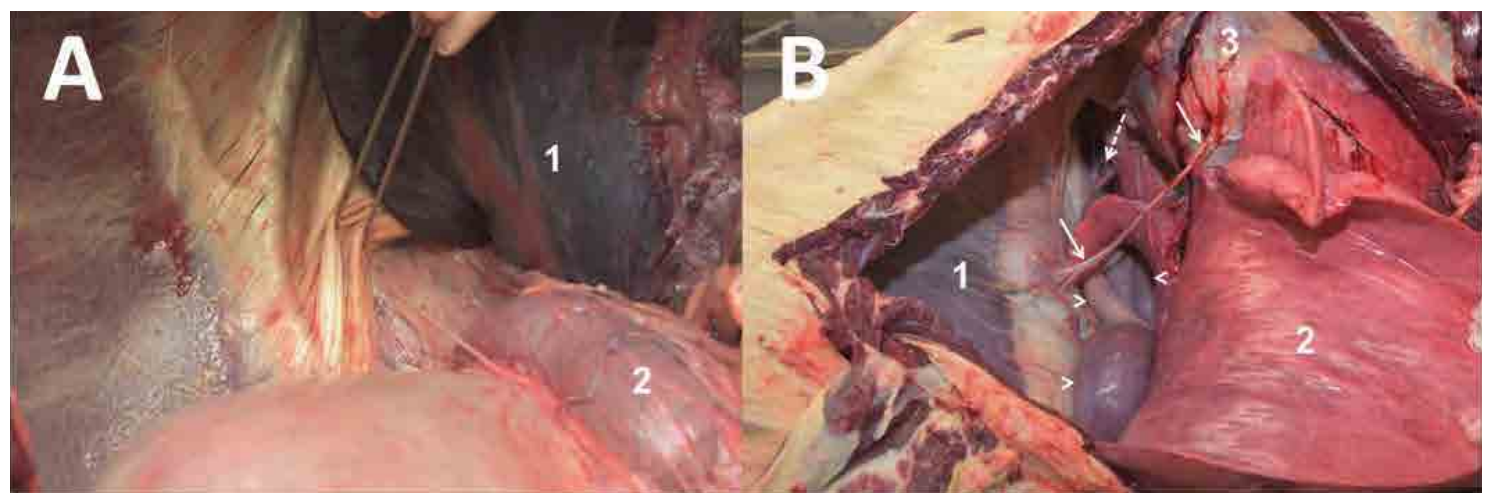

Ultrasound diagnosis of a diaphragmatic hernia in a 23-year-old Hanoverian gelding

Fig. 3: Gross pathology, view from caudal, horse in dorsal recumbency. A) The ventral border of the diaphragmatic tear is held by the forceps. Part of liver (1) and small intestine (2) are herniating into the thoracic cavity through the diaphragmatic tear. B) Diaphragm (1), lung (2) and pericardium with heart (3) are identified. Distended and ingesta-filled small intestinal loops (arrowheads) are lying between the diaphragm and the lung lobe. Most of the mediastinum is no longer present with only the phrenic nerve (arrows) and small amounts of mesh like mediastinal structure (dashed arrow) visible.

and financial reasons. As the horse was stable, the decision was made to refeed and monitor for signs of abdominal pain.

\section{Treatment and Outcome}

Over the next 3 days the horse remained stable with no signs of abdominal pain. Daily ultrasounds were unchanged from the previous examination. On the fourth day the horse showed signs of acute, severe abdominal pain. On ultrasound, both small and large intestinal loops were visible within the thorax. In addition, distended small intestine (diameter $>4 \mathrm{~cm}$ ) was observed in the right and left ventral inguinal regions, indicating obstruction to the movement of ingesta. When the abdominal pain failed to improve with medical therapy (flunixin meglumine $1.1 \mathrm{mg} / \mathrm{kg}$ bwt I.V., Fluniximin, Dr. E. Graeub AG, Bern, Switzerland) the owner requested the horse be euthanized.

\section{Pathologic Findings}

At necropsy, a diaphragmatic tear, $15 \mathrm{~cm}$ in length, was located in the right dorsal quadrant directly on the right side of the vena cava (Fig. 3A). The ventral border of the hernial ring consisted of the central tendon and the dorsal border was the fibrotic thickened muscular part of the diaphragm. Seven meters of small intestine (ileum and the caudal part of the jejunum) and the caecal base were located within the thoracic cavity. The right liver lobe was displaced and firmly attached to the pleural surface of the right thoracic wall. The mediastinum was no longer present with only the phrenic nerve and small amounts of mesh like mediastinal tissue visible on both sides (Fig. 3B).

\section{Discussion}

This case report illustrates the usefulness of ultrasonographic examination of the thorax and abdomen in the clinical assessment of a horse with a diaphragmatic hernia and highlights the benefit of repeated ultrasonographic examinations, especially if a definitive diagnosis is unable to be reached on the initial evaluation. It also emphasizes the need to consider the diaphragm and thoracic cavity when evaluating horses with abdominal pain and weight loss. As a result of improved technology, training and the availability of appropriate equipment, abdominal ultrasonography has become central to the diagnostic evaluation in many different case presentations, i.e. colic, weight loss, fever of unknown origin (Henry Barton, 2011; le Jeune and Whitcomb, 2014). As a full examination of the entire abdomen is not always possible in emergency situations, focused or fast localized abdominal sonography (FLASH) techniques have been recently reported for use when evaluating horses with acute colic (Busoni et al., 2011). It is recommended to include at least one thoracic window in the protocol to assess for the presence of pleural fluid and abdominal organs even in the abbreviated examination of an emergency patient.

A complete abdominal ultrasonographic evaluation should include assessment of the diaphragm on both sides (Morresey, 2014).The diaphragm of the horse is described as being recognizable on ultrasound by its parallel echogenic fascial planes with lucent musculature between. The curved shape of the diaphragm, air filled lung and ultrasonographic beam geometry make complete visualization unlikely (Rantanen, 1986). Even so, this case report reinforces that ultrasonographic proof of a disruption of the diaphragmatic line (discontinuity of the diaphragm, thickening and wavy appearance of the diaphragm distally) is possible. 
Ultrasound diagnosis of a diaphragmatic hernia in a 23-year-old Hanoverian gelding

G. Kaupp, K.J. Mitchell
In the case reported here, thoracic radiography did not aid the diagnosis which is consistent with other reports (Hartzband et al., 1990; Goehring et al., 1999; Hart and Brown, 2009). Although radiography might reveal displacement of gas or ingesta filled intestine, diagnostic thoracic radiography in colicky adult horses is difficult to perform in the field setting. Rectal examination is a mainstay of the diagnostic evaluation in horses with colic. However, horses with diaphragmatic hernias do not always have abnormal rectal findings and the feeling of 'emptiness' that is occasionally reported should not be relied upon (Hart and Brown, 2009; Romero and Rodgerson, 2010).

Diaphragmatic rent and visceral herniation carries a poor prognosis for survival. Most horses are euthanized after the diagnosis is made, as surgical repair of the defect is extremely technically challenging (Hart and Brown, 2009; Romero and Rodgerson, 2010). This report of a chronic diaphragmatic tear with intermittent visceral herniation shows the value of repeated ultra-

\section{References}

Busoni, V., De Busscher, V., Lopez, D.: Evaluation of a protocol for fast localised abdominal sonography of horses (FLASH) admitted for colic. Vet. J. 2011, 188: 77-82.

Goehring, L. S., Goodrich, L. R., Murray, M. J.: Tachypnoea associated with a diaphragmatic tear in a horse. Equine veterinary journal 1999, 31: 443-445.

Hart, S. K., Brown, J. A.: Diaphragmatic hernia in horses: 44 cases (1986-2006). J. Vet. Emerg. Crit. Care. 2009 (San Antonio) 19: 357-362.

Hartzband, L. E., Kerr, D. V., Morris, E. A.: Ultrasonographic diagnosis of diaphragmatic rupture in a horse. Veterinary Radiology 1990, 31: 42-44.

Hassel, D. M.: Thoracic trauma in horses. Vet. Clin. North. Am. Equine. Pract. 2007, 23: 67-80.

Henry Barton, M.: (2011) Understanding abdominal ultrasonography in horses: which way is up? Compendium (Yardley, PA) 2011, 33: E2.

le Jeune, S., Whitcomb, M. B.: Ultrasound of the Equine Acute Abdomen. Vet. Clin. North Am. Equine Practi. 2014, 30: 353-381.

Morresey, P. R.: Ultrasonography of the pleural cavity, lung and diaphragm. In: Atlas of equine ultrasonography. John Wiley \& Sons, Inc., Chichester, West Sussex, 2014, 367-378.

Rantanen, N. W.: Diseases of the thorax. Vet. Clin. North. Am. Equine. Pract. 1986, 2: 49-66.

Romero, A.E., Rodgerson, D. H.: Diaphragmatic herniation in the horse: 31 cases from 2001-2006. Canadi. Vet. J. 2010, 51: 1247-1250.

Williams, J., Leveille, R., Myer, C. W.: Imaging modalities used to confirm diaphragmatic hernia in small animals. Compend. Contin. Educ. Pract. Vet. 1998, 20: 1199-1210. sonographic evaluation of the thorax and diaphragm to diagnose and monitor the hernia, in the face of non-diagnostic thoracic radiography and rectal exam findings. Ultrasonographic evaluation can be easily performed in the field setting with readily available equipment. Achieving a pre-surgical ante mortem diagnosis can reduce costs and provide prognostic information for both clinicians and owners.

\section{Acknowledgements}

The authors would like to thank Prof. Dr. med. vet. Colin C. Schwarzwald for the pictures taken at necropsy.

\section{Conflict of interest:}

The authors have no conflict of interest to declare.

\section{Corresponding author}

Katharyn Jean Mitchell

BVSc, DipVetClinStud, Diplomate ACVIM (LAIM)

Universität Zürich

Vetsuisse-Fakultät Departement für Pferde

Klinik für Pferdemedizin Büro TFA 10.37

Winterthurerstrasse 260

$\mathrm{CH}-8057$ Zürich

Tel. 0041 (0) 446359112

Fax 0041 (0) 446358905

E-Mail:kmitchell@vetclinics.uzh.ch 